\title{
Nonautonomous Discrete Neuron Model with Multiple Periodic and Eventually Periodic Solutions
}

\author{
Alexander N. Pisarchik, ${ }^{1,2}$ Michael A. Radin, ${ }^{3}$ and Ryan Vogt ${ }^{3}$ \\ ${ }^{1}$ Centro de Investigaciones en Optica, Loma del Bosque 115, Lomas del Campestre, 37150 León, GTO, Mexico \\ ${ }^{2}$ Center for Biomedical Technology, Technical University of Madrid, Campus de Montegancedo, 28223 Madrid, Spain \\ ${ }^{3}$ College of Science, Rochester Institute of Technology, 1 Lomb Memorial Drive, Rochester, NY 14623, USA
}

Correspondence should be addressed to Michael A. Radin; michael.radin@rit.edu

Received 20 April 2015; Accepted 25 May 2015

Academic Editor: Lu Zhen

Copyright (C) 2015 Alexander N. Pisarchik et al. This is an open access article distributed under the Creative Commons Attribution License, which permits unrestricted use, distribution, and reproduction in any medium, provided the original work is properly cited.

We introduce a nonautonomous discrete neuron model based on the Rulkov map and investigate its dynamics. Using both the linear stability and bifurcation analyses of the system of piecewise difference equations, we determine dynamical bifurcations and parameter regions of steady-state and periodic solutions.

\section{Introduction}

Piecewise difference equations exhibit very rich dynamics because the lack of differentiability makes their solutions either eventually constant, eventually periodic of various periods, or eventually chaotic [1]. The $3 n+1$ conjecture proposed by Lothar Collatz in 1937 and the tent map were the first considered piecewise linear difference equations $[2,3]$. Later, piecewise difference equations have been used as mathematical models for various applications, including neurons (for extensive review, see [4] and references therein).

In this paper, we focus on the Rulkov map as one of the simplest systems to model neuron dynamics [5]. The original Rulkov map is the autonomous system which reproduces the spiking behavior similar to biological neurons. Although this model does not have real parameters, it is computationally less costly than neurophysiological models, such as, the Hodgkin-Huxley model [6], and hence it can be easily used for simulation of a complex network of synaptically coupled neurons. Being a part of the complex neural network, a neuron is not separated; its dynamics is affected by oscillations of the neighboring neurons through synapses. Therefore, the neuron can be considered as a nonautonomous system.

\section{Model}

The autonomous Rulkov map is the system of piecewise difference equations that consists of three components $[5,7]$ :

$$
\begin{array}{r}
x_{n+1}=\frac{\alpha}{1-x_{n}}+y_{n}, \\
y_{n+1}=y_{n}-\mu\left(x_{n}+1\right)+\mu \sigma, \\
n=0,1, \ldots,
\end{array}
$$

when $x_{n} \leq 0$,

$$
\begin{aligned}
& x_{n+1}=\alpha+y_{n}, \\
& y_{n+1}=y_{n}-\mu\left(x_{n}+1\right)+\mu \sigma, \\
& n=0,1, \ldots,
\end{aligned}
$$

when $x_{n} \leq \alpha+y_{n}$ and $x_{n-1} \leq 0$, and

$$
\begin{aligned}
x_{n+1} & =-1, \\
y_{n+1} & =y_{n}-\mu\left(x_{n}+1\right)+\mu \sigma, \\
n & =0,1, \ldots,
\end{aligned}
$$

when $x_{n}>\alpha+y_{n}$ or $x_{n-1}>0$. 
To convert the autonomous Rulkov map equations (1)(3) into a nonautonomous system, we introduce two periodic parameters $\alpha_{0}$ and $\alpha_{1}$ as follows:

$$
\alpha_{n}= \begin{cases}\alpha_{0}, & \text { if } n \text { is even, } \\ \alpha_{1}, & \text { if } n \text { is odd }\end{cases}
$$

It is our goal to investigate monotonic, periodic, and chaotic characters of solutions. We will start with a linear stability analysis of equilibrium points of the autonomous system equations (1)-(5).

\section{Linear Stability Analysis of the Autonomous System}

We will analyze the local stability of the equilibrium points of the autonomous map linearizing each of the three components individually. The first component is the following system:

$$
\begin{aligned}
& x_{n+1}=\frac{\alpha}{1-x_{n}}+y_{n}, \\
& y_{n+1}=y_{n}-\mu\left(x_{n}+1\right)+\mu \sigma, \\
& \quad n=0,1, \ldots,
\end{aligned}
$$

when $x_{n} \leq 0$. By setting

$$
\begin{aligned}
& \bar{x}=\frac{\alpha}{1-\bar{x}}+\bar{y}, \\
& \bar{y}=\bar{y}-\mu(\bar{x}+1)+\mu \sigma,
\end{aligned}
$$

we get the following equilibrium point:

$$
\begin{aligned}
& \bar{x}=\sigma-1, \\
& \bar{y}=\sigma-1-\frac{\alpha}{2-\sigma} .
\end{aligned}
$$

Now, let

$$
\begin{aligned}
& f(x, y)=\frac{\alpha}{1-x}+y, \\
& g(x, y)=y-\mu(x+1)+\mu \sigma .
\end{aligned}
$$

Then,

$$
\begin{aligned}
& f_{x}(x, y)=\frac{\alpha}{(1-x)^{2}} \\
& f_{y}(x, y)=1, \\
& g_{x}(x, y)=-\mu, \\
& g_{y}(x, y)=1 .
\end{aligned}
$$

So, we get the following Jacobian matrix $J_{1}$ :

$$
\begin{aligned}
J_{1} & =\left[\begin{array}{cc}
f_{x}(\bar{x}, \bar{y}) & f_{y}(\bar{x}, \bar{y}) \\
g_{x}(\bar{x}, \bar{y}) & g_{y}(\bar{x}, \bar{y})
\end{array}\right]=\left[\begin{array}{cc}
\frac{\alpha}{(1-(\sigma-1))^{2}} & 1 \\
-\mu & 1
\end{array}\right] \\
& =\left[\begin{array}{cc}
\frac{\alpha}{(2-\sigma)^{2}} & 1 \\
-\mu & 1
\end{array}\right] .
\end{aligned}
$$

Thus, the eigenvalues of $J_{1}$ are the roots of the following characteristic polynomial:

$$
\begin{aligned}
& \left(\lambda-\frac{\alpha}{(2-\sigma)^{2}}\right)(\lambda-1)+\mu \\
& \quad=\lambda^{2}-\lambda\left[1+\frac{\alpha}{(2-\sigma)^{2}}\right]+\left[\frac{\alpha}{(2-\sigma)^{2}}+\mu\right]=0 .
\end{aligned}
$$

Hence, we see that $\left|\lambda_{1}\right|<1$ and $\left|\lambda_{2}\right|<1$ of $J_{1}$ if and only if

$$
1+\frac{\alpha}{(2-\sigma)^{2}}<\frac{\alpha}{(2-\sigma)^{2}}+\mu+1<2
$$

and if and only if

(i)

$$
\alpha<(2-\sigma)^{2}(1-\mu)
$$

(ii)

$$
\sigma \neq 2
$$

$$
\mu<1
$$

Next, we will analyze the local stability of the equilibrium points of the second component of the autonomous system:

$$
\begin{aligned}
& x_{n+1}=\alpha+y_{n}, \\
& y_{n+1}=y_{n}-\mu\left(x_{n}+1\right)+\mu \sigma, \\
& n=0,1, \ldots,
\end{aligned}
$$

when $x_{n} \leq \alpha+y_{n}$ and $x_{n-1} \leq 0$. By setting

$$
\begin{aligned}
& \bar{x}=\alpha+\bar{y} \\
& \bar{y}=\bar{y}-\mu(\bar{x}+1)+\mu \sigma,
\end{aligned}
$$

we get the following equilibrium point:

$$
\begin{aligned}
& \bar{x}=\sigma-1, \\
& \bar{y}=\sigma-1-\alpha .
\end{aligned}
$$

Now, let

$$
\begin{aligned}
& f(x, y)=\alpha+y, \\
& g(x, y)=y-\mu(x+1)+\mu \sigma .
\end{aligned}
$$

Then,

$$
\begin{aligned}
& f_{x}(x, y)=0, \\
& f_{y}(x, y)=1, \\
& g_{x}(x, y)=-\mu, \\
& g_{y}(x, y)=1 .
\end{aligned}
$$


So, we get the following Jacobian matrix $J_{2}$ :

$$
J_{2}=\left[\begin{array}{ll}
f_{x}(\bar{x}, \bar{y}) & f_{y}(\bar{x}, \bar{y}) \\
g_{x}(\bar{x}, \bar{y}) & g_{y}(\bar{x}, \bar{y})
\end{array}\right]=\left[\begin{array}{cc}
0 & 1 \\
-\mu & 1
\end{array}\right] .
$$

Thus, the eigenvalues of $J_{2}$ are the roots of the following characteristic polynomial:

$$
(\lambda-0)(\lambda-1)+\mu=\lambda^{2}-\lambda+\mu=0 .
$$

Hence, we see that $\left|\lambda_{1}\right|<1$ and $\left|\lambda_{2}\right|<1$ of $J_{2}$ if and only if

$$
1<1+\mu<2
$$

and if and only if

$$
\mu<1 \text {. }
$$

Finally, we will analyze the local stability of the equilibrium points of the third component of the autonomous system:

$$
\begin{aligned}
& x_{n+1}=-1, \\
& y_{n+1}=y_{n}-\mu\left(x_{n}+1\right)+\mu \sigma, \\
& \quad n=0,1, \ldots,
\end{aligned}
$$

when $x_{n}>\alpha+y_{n}$ or $x_{n-1}>0$. By setting

$$
\begin{aligned}
& \bar{x}=-1, \\
& \bar{y}=\bar{y}-\mu(\bar{x}+1)+\mu \sigma,
\end{aligned}
$$

we get the following equilibrium point:

$$
\begin{aligned}
& \bar{x}=-1, \\
& \bar{y}=\Re .
\end{aligned}
$$

Now, let

$$
\begin{aligned}
& f(x, y)=\alpha+y, \\
& g(x, y)=y-\mu(x+1)+\mu \sigma .
\end{aligned}
$$

Then,

$$
\begin{aligned}
& f_{x}(x, y)=0, \\
& f_{y}(x, y)=0, \\
& g_{x}(x, y)=-\mu, \\
& g_{y}(x, y)=1 .
\end{aligned}
$$

So, we get the following Jacobian matrix $J_{3}$ :

$$
J_{2}=\left[\begin{array}{ll}
f_{x}(\bar{x}, \bar{y}) & f_{y}(\bar{x}, \bar{y}) \\
g_{x}(\bar{x}, \bar{y}) & g_{y}(\bar{x}, \bar{y})
\end{array}\right]=\left[\begin{array}{cc}
0 & 0 \\
-\mu & 1
\end{array}\right] .
$$

Thus, the eigenvalues of $J_{3}$ are $\lambda_{1}=0$ and $\lambda_{2}=0$.

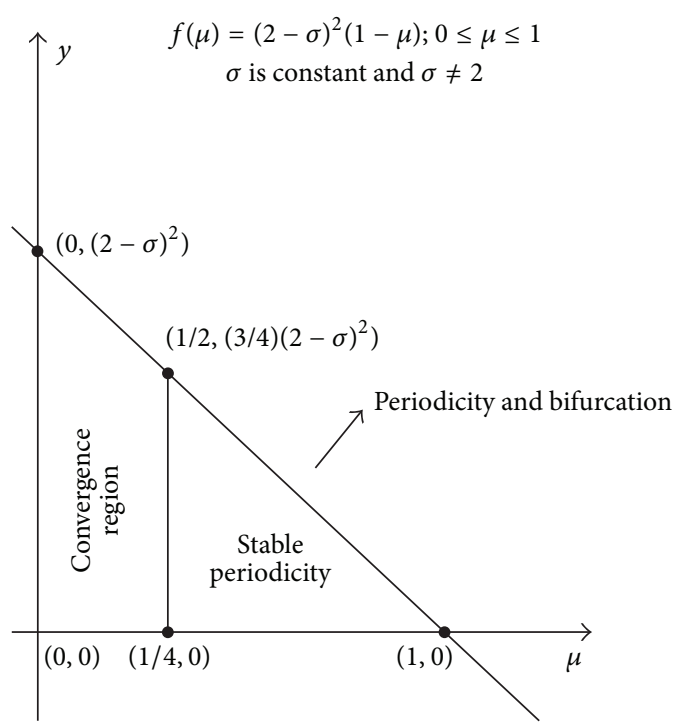

FIGURE 1: Bifurcation diagram with $\mu$ as a control parameter for $\sigma=$ 1.

\section{Stability of the Nonautonomous System}

From the linearized stability analysis, we decompose the nonautonomous system into six components and apply the linearized stability analysis on each one as previously done from which we obtain the following stability conditions:

(1) stability conditions:

$$
\alpha \leq(2-\sigma)^{2}(1-\mu), \quad \mu \leq \frac{1}{4},
$$

(2) stable periodic conditions:

$$
\alpha_{1} \leq(2-\sigma)^{2}(1-\mu), \quad \frac{1}{4}<\mu<1 .
$$

These conditions result in two bifurcation diagrams shown in Figures 1 and 2. By letting $\sigma=1$ be constant, we get the straight line which bounds different stability regions.

Next, by letting $\mu=0.2$ be constant, we get the parabola shown in Figure 2, which bounds different stability regions.

From the linear stability analysis of the autonomous systems and from the two bifurcation diagrams shown in Figures 1 and 2, we obtain the following stability conditions:

(i) stability conditions:

$$
\max \left\{\alpha_{0}, \alpha_{1}\right\} \leq(2-\sigma)^{2}(1-\mu), \quad \mu \leq \frac{1}{4}
$$

(ii) stable periodic conditions:

$$
\max \left\{\alpha_{0}, \alpha_{1}\right\} \leq(2-\sigma)^{2}(1-\mu), \quad \frac{1}{4}<\mu<1,
$$

(iii) instability and bifurcations

$$
\min \left\{\alpha_{0}, \alpha_{1}\right\}>(2-\sigma)^{2}(1-\mu) .
$$




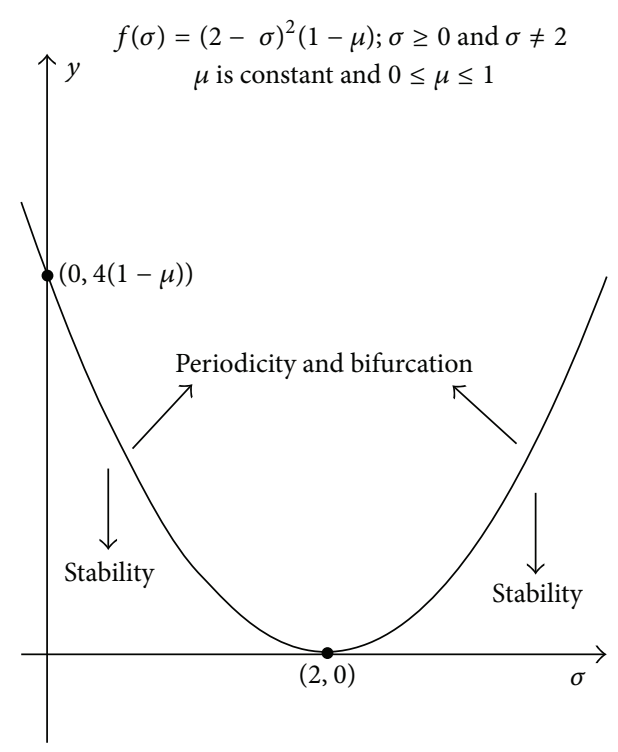

FIGURE 2: Bifurcation diagram with $\sigma$ as a control parameter for $\mu=$ 0.2 .

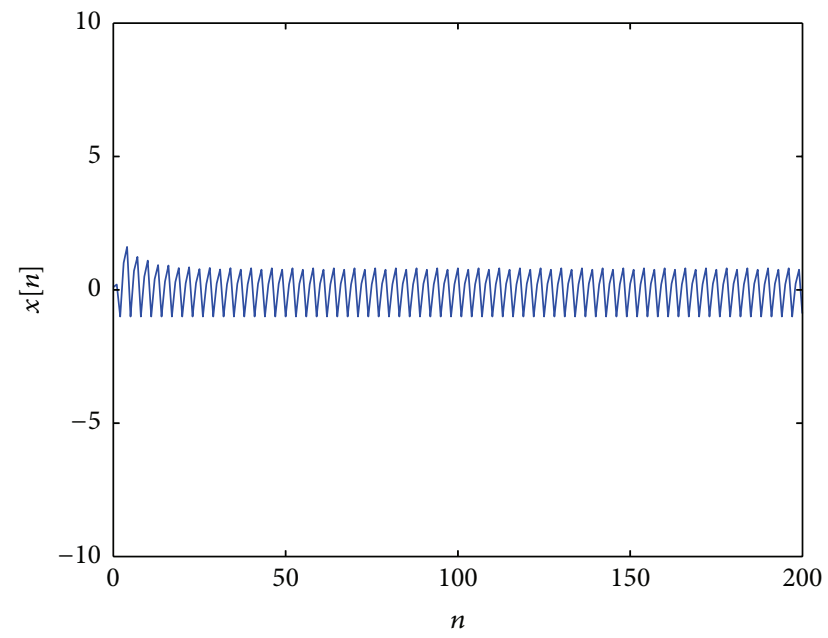

FIGURE 3: Eventually steady periodic cycle satisfies condition (i).

\section{Time Series}

To illustrate the map dynamics, we will present some graphical examples for various parameters in different regions of the stability diagrams shown in Figures 1 and 2.

Example 1. In this example, we assume that condition (i) mentioned above is satisfied, where $\mu=0.2, \sigma=1, \alpha_{0}=0.7$, and $\alpha_{1}=0.75$. Then, we obtain an eventually steady periodic cycle in Figure 3.

We see that in this case the solution becomes eventually periodic.

Example 2. In this example, we assume that (ii) is satisfied, where $\mu=0.5, \sigma=1, \alpha_{0}=0.4$, and $\alpha_{1}=0.3$. This gives us the periodic orbit shown in Figure 4.

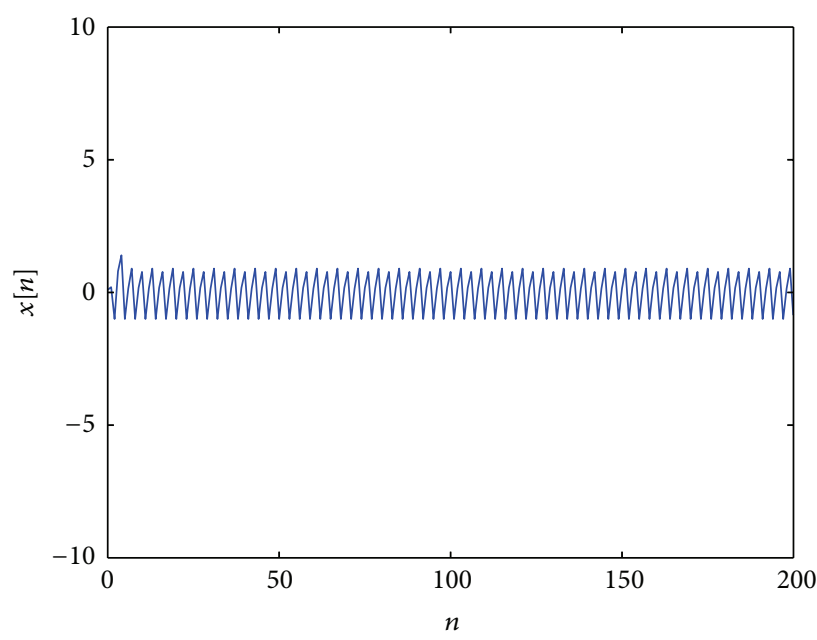

FIgURe 4: Periodic orbit satisfies condition (i).

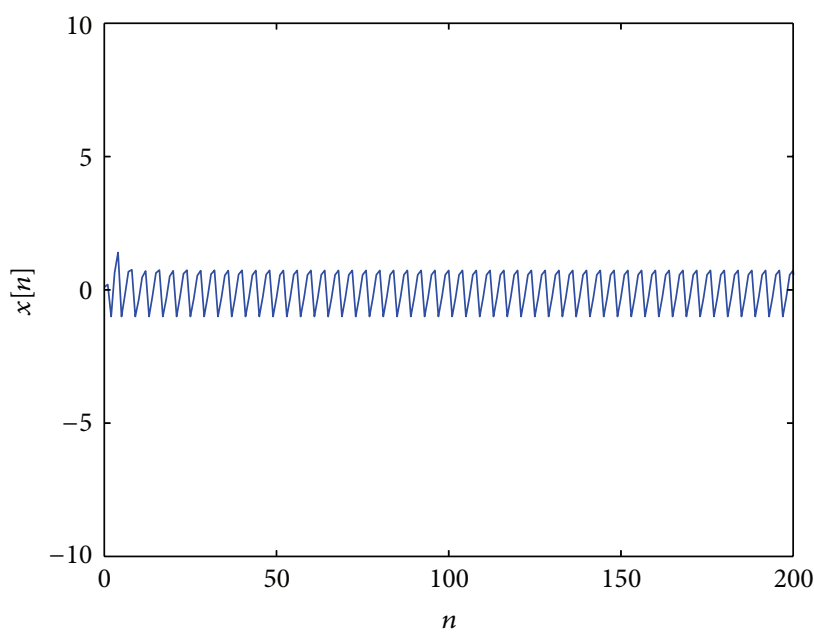

FIgure 5: Periodic orbit satisfies condition (i).

Now, we observe that even though we are in the stability region, the solution is eventually periodic instead of being eventually constant as we have complex eigenvalues when $\mu>1 / 4$.

Example 3. In this example, we assume that (ii) is satisfied, where $\mu=0.75, \sigma=1, \alpha_{0}=0.2$, and $\alpha_{1}=0.1$, which gives us the graph in Figure 5.

Now, we observe that the periodic character of the solutions changes as we have complex eigenvalues when $\mu>$ $1 / 4$.

Example 4. In this example, we assume that (ii) fails by letting $\mu=0.75, \sigma=0.1, \alpha_{0}=1.5$, and $\alpha_{1}=0.75$ which gives us the graph in Figure 6.

Notice that this is eventually periodic orbit with a different period compared to Example 4 due to the fact that the stability conditions fail. 


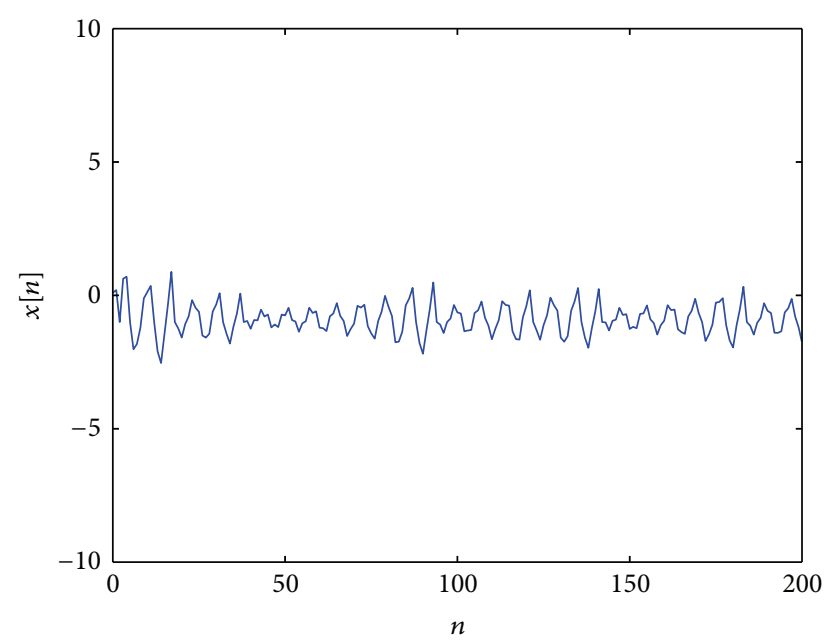

FIgURE 6: Chaotic orbit satisfies condition (ii).

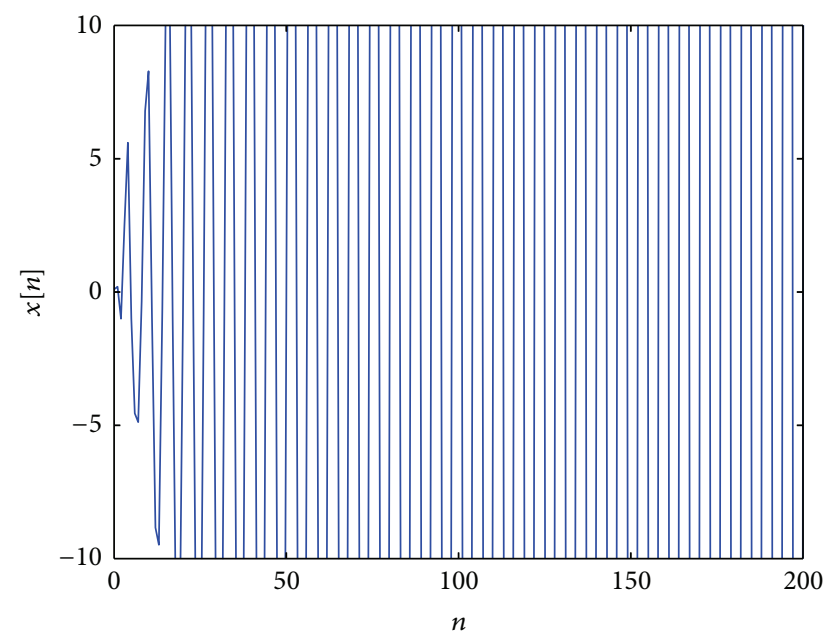

FIGURE 7: Unbounded solutions satisfy condition (ii).

Example 5. In this example, we will assume that (ii) fails by letting $\mu=1, \sigma=1, \alpha_{0}=4$, and $\alpha_{1}=4.1$ which gives us the graph in Figure 7.

Notice that the periodicity is quite substantially different compared to the previous examples due to the fact that the stability conditions fail.

Example 6. In this example, we will assume that (ii) fails by letting $\mu=1, \sigma=1, \alpha_{0}=0.4$, and $\alpha_{1}=0.5$ which gives us the graph in Figure 8.

Notice that the periodicity is quite substantially different compared to the previous examples due to the fact that the stability conditions fail. Furthermore, we observe that when $\mu>1 / 4$, the periodic character of the solutions changes, unstable periodic orbits appear, and chaotic behavior appears as well.

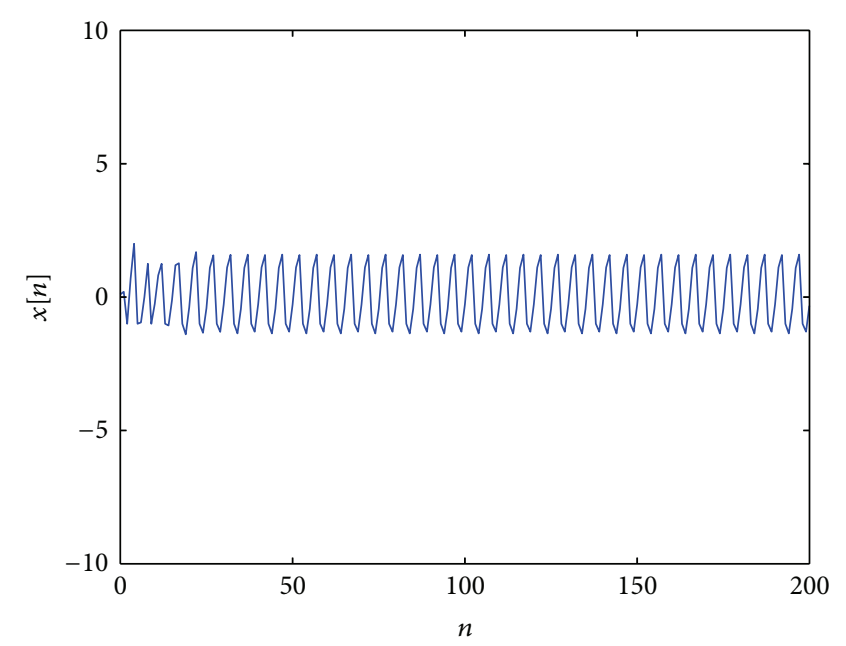

FIGURE 8: Eventually periodic orbit satisfies condition (ii).

\section{Conclusions and Future Works}

On the base of an autonomous Rulkov map, we designed a nonautonomous discrete neuron model. We demonstrated steady state, periodic, and chaotic character of solutions and performed a linear stability analysis of the equilibrium points of both the autonomous and nonautonomous Rulkov maps.

Our future goal is to generalize the results when $\left\{\alpha_{n}\right\}_{n=0}^{\infty}$ is periodic with period $p \geq 3$. In particular, it would be interesting to compare similarities and differences that will arise with the results of this paper when $\left\{\alpha_{n}\right\}_{n=0}^{\infty}$ is periodic with period 2. Furthermore, it would be of paramount interest to introduce the periodic parameter $\left\{\sigma_{n}\right\}_{n=0}^{\infty}$ to increase the interaction between the neurons of the model and make the model much more accurate and, moreover, to show how the interaction between the terms of $\left\{\alpha_{n}\right\}_{n=0}^{\infty}$ and $\left\{\sigma_{n}\right\}_{n=0}^{\infty}$ determines the stability of solutions, periods of solutions, and boundedness of solutions.

\section{Conflict of Interests}

The authors declare that there is no conflict of interests regarding the publication of this paper.

\section{Acknowledgment}

This study was supported by the BBVA-UPM Isaac Peral BioTech Program.

\section{References}

[1] V. C. Carmona, E. Freire, E. Ponce, and F. Torres, "On simplifying and classifying piecewise-linear systems," IEEE Transactions on Circuits and Systems I: Fundamental Theory and Applications, vol. 49, no. 5, pp. 609-620, 2002.

[2] D. L. Johnson and C. D. Maddux, Logo: A Retrospective, Haworth Press, New York, NY, USA, 1997.

[3] J. Gleich, Chaos: The Amazing Science of the Unpredictable, Vintage Books, London, UK, 1998. 
[4] B. Ibarz, J. M. Casado, and M. A. F. Sanjuán, "Map-based models in neuronal dynamics," Physics Reports, vol. 501, no. 1-2, pp. 174, 2011.

[5] N. F. Rulkov, "Modeling of spiking-bursting neural behavior using two-dimensional map," Physical Review E, vol. 65, no. 4, Article ID 041922, 2002.

[6] A. L. Hodgkin and A. F. Huxley, "A quantitative description of membrane current and its application to conduction and excitation in nerve," The Journal of Physiology, vol. 117, no. 4, pp. 500-544, 1952.

[7] J. M. Sausedo-Solorio and A. Pisarchik, "Synchronization of map-based neurons with memory and synaptic delay," Physics Letters A, vol. 378, no. 30-31, pp. 2108-2112, 2014. 


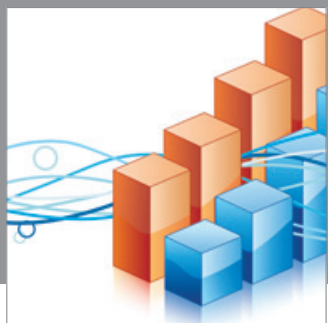

Advances in

Operations Research

mansans

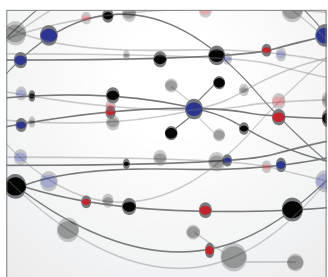

The Scientific World Journal
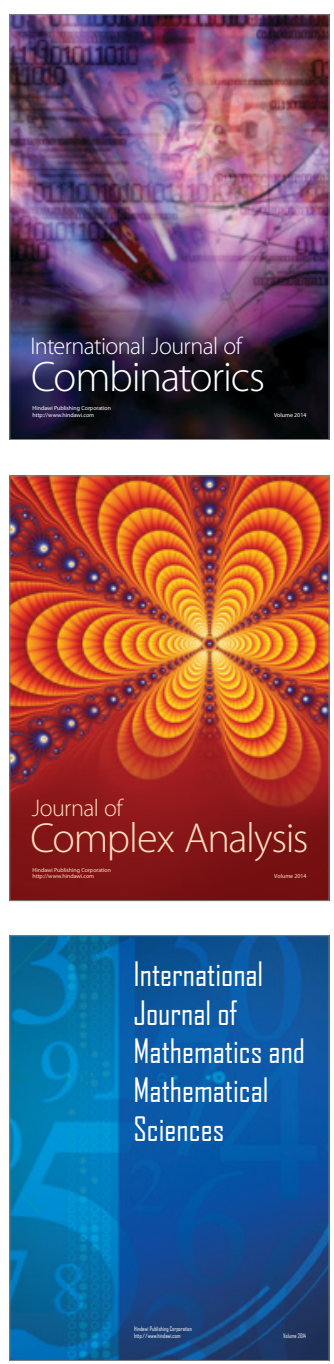
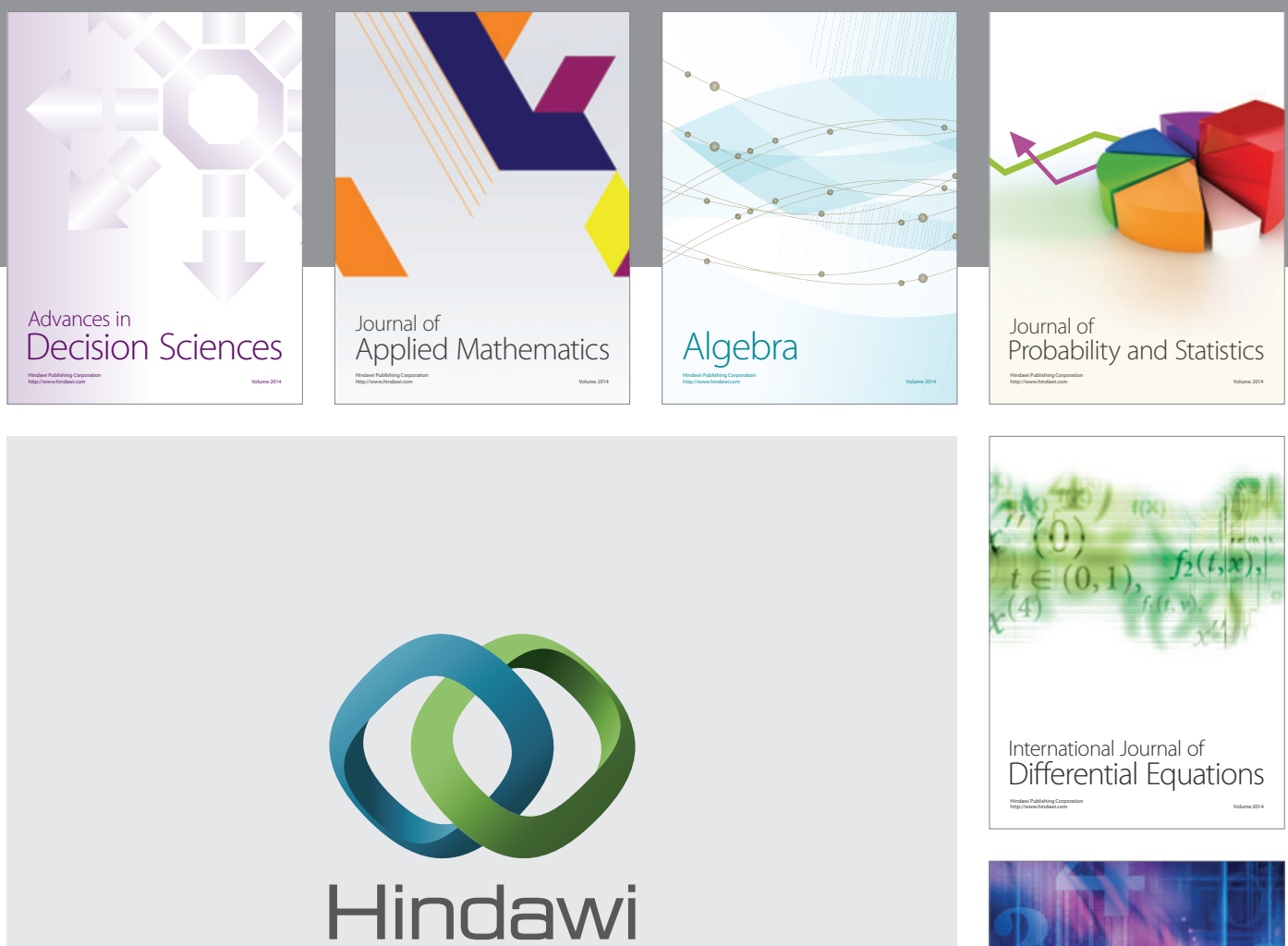

Submit your manuscripts at http://www.hindawi.com
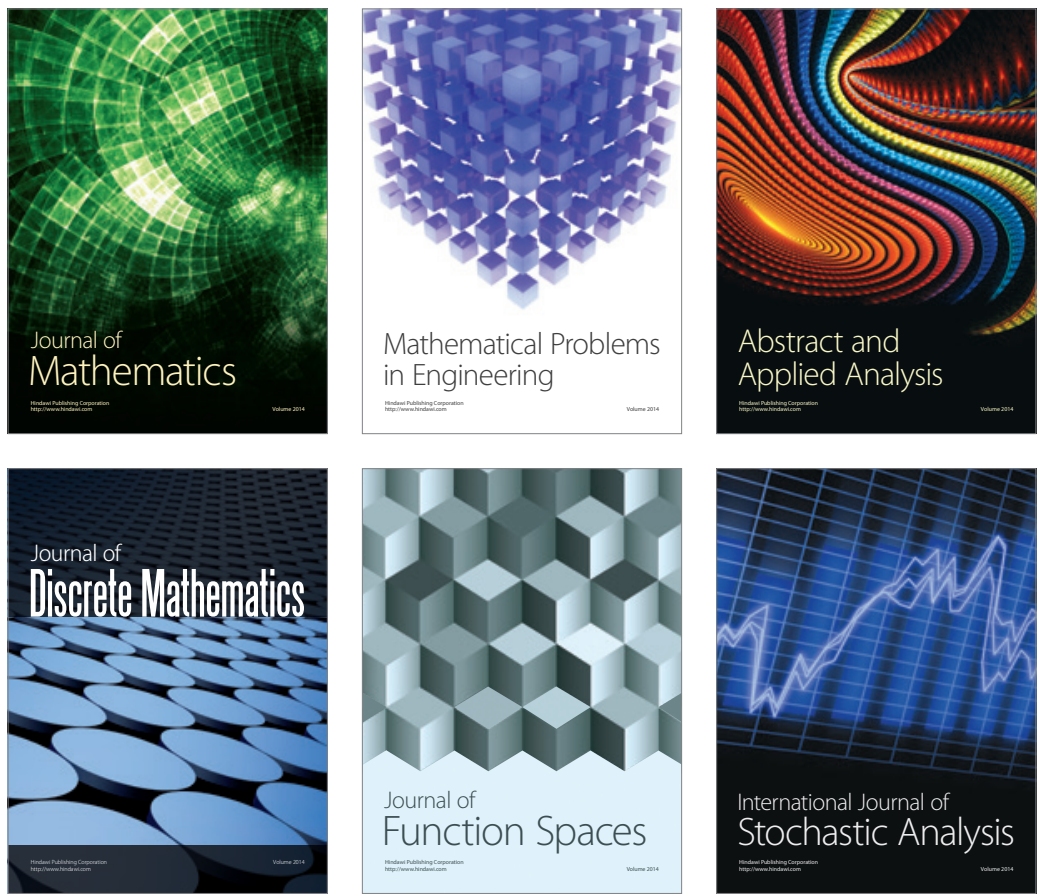

Journal of

Function Spaces

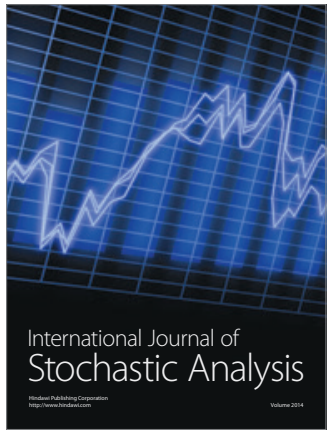

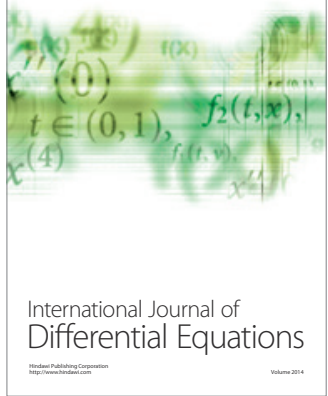
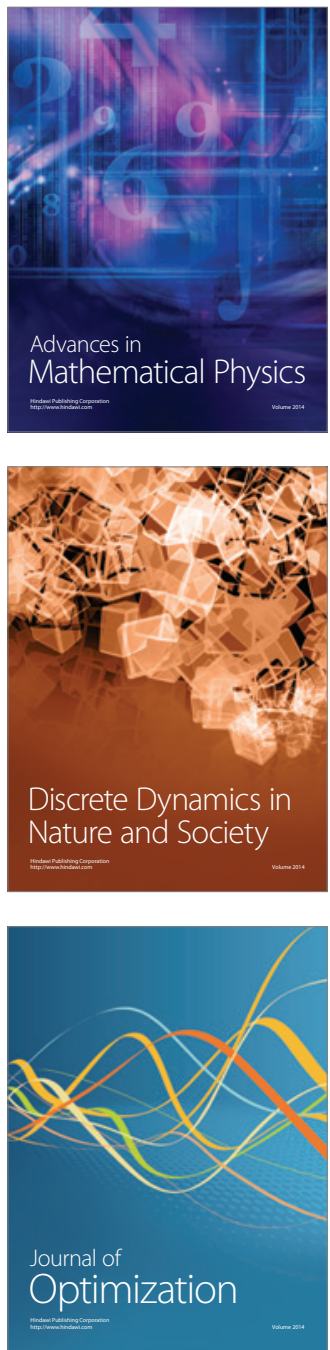